\title{
INTELIGÊNCIA ARTIFICIAL E TEORIA DE RESOLUÇÃO DE PROBLEMAS
}

\author{
João de Fernandes TEIXEIRA* \\ Maria Eunice QUILICI GONZALES*
}

\begin{abstract}
RESUMO: Este artigo é uma tentativa de delinear as principais características da pesquisa numa nova área de estudos a chamada Inteligência Artificial (AI). Os itens 1 e 2 constituem um rápido histórico da AI e seus pressupostos básicos. O item 3 trata da teoria de resolução de problemas, desenvolvida por $A$. Newell e H. Simon. O item 4 procura mostrar a relevância da AI para a Filosofia, em especial para a Filosofia da Mente e para a Teoria do Conhecimento.

UNITERMOS: Inteligência artificial; filosofia da mente; métodos de resolução de problemas; simulação cognitiva.
\end{abstract}

\section{Introdução}

Quando A. Turing no seu artigo "Computing Machinery and Intelligence" (1950) (16) levantou a questão de se é possível para uma máquina pensar, ele imediatamente considerou a necessidade de definir o termo pensar. Diante da complexidade desta tarefa, Turing propôs que uma tal questão fosse substituída por uma outra que lhe parecia bastante próxima da questão original. Essa questão consistia em saber se é possível para uma máquina imitar o comportamento humano inteligente, com um grau de perf eição tão alto, de tal forma que um juiz humano pudesse ser confundido ao ter que decidir, com base nos resultados das tarefas realizadas, se tais tarefas foram executadas por um homem ou por uma máquina. No mesmo artigo de 1950, Turing esboça uma proposta de pesquisa que parecia tornar possível uma resposta afirmativa a sua última questão.

A proposta de Turing reapareceu em 1961 no artigo de A. Newell e H. Simon -
"The Simulation of Human Thought" (10) - na condição de teste de uma teoria de resolução humana de problemas. Esta teoria foi elaborada por estes autores para explicar alguns aspectos dos processos mentais responsáveis pelo comportamento humano inteligente. Ela constitui uma amostra dos primeiros trabalhos desenvolvidos por um grupo de pesquisadores que, juntamente com Newell e Simon, organizaram um projeto de estudos que ficou sendo conhecido pelo nome de Projeto de Simulação Cognitiva. Tal projeto enquadra-se em uma área de pesquisas mais ampla denominada Inteligência Artificial.

No que se segue pretendemos apresentar, de forma bastante simplificada, alguns tópicos tratados na área de Inteligência Artificial que são de interesse para a Filosofia e para a Psicologia. De modo a delimitar nosso campo de discussão, começaremos por fornecer algumas noções básicas daquilo que se entende por Inteligência Artificial. Num segundo momento, apresen-

* Departamento de Filosofia - Faculdade de Educação, Ciências Sociais e da Documentação - UNESP — 17.500 Marilia - SP. 
TEIXEIRA, J. de F. \& QUILICI GONZALES, M.E. — Inteligência Artificial e teoria de resolução de problemas. Trans/Form/Ação, São Paulo, 6: 45-52, 1983.

taremos as principais diretrizes do projeto original de Newell e Simon que pode ser resumido na proposta de simular a atividade de resolução humana de problemas através do computador digital. Finalizaremos com uma amostragem de reflexões recentes acerca da Inteligência Artificial, comentando algumas questões que a vinculam à Filosofia, em especial à Filosofia da Mente e à Teoria do Conhecimento.

\section{Noções Preliminares de Inteligência Ar- tificial}

A Inteligência Artificial, no sentido genérico do termo (daqui para frente $\mathrm{AI}$, do inglês Artificial Intelligence) é uma área de estudos ainda não muito bem delimitada que reúne a Ciência da Computação, a Psicologia, a Lingüística e a Filosofia. Ela possui duas vertentes principais representadas pelo Projeto de Simulação Cognitiva e pelo Projeto de Inteligência Artificial num sentido restrito do termo.

A distinção entre os dois projetos reside, aparentemente, no grau de realidade psicológica dos modelos elaborados pelos pesquisadores nos seus respectivos domínios de estudo. O Projeto de Simulação Cognitiva tem como objeto de estudos o comportamento humano. O objetivo dos seus pesquisadores é estudar e explicar os processos mentais humanos responsáveis pelo comportamento inteligente. $\mathrm{O}$ estudo desses processos é feito com o auxílio de técnicas computacionais que permitem a construção de modelos, na forma de programas, para a simulação de segmentos do comportamento humano, como por exemplo, a atividade de resolução de problemas.

No caso do projeto de Inteligência Artificial, no sentido restrito do termo, existe uma preocupação menor por parte dos pesquisadores, com o grau de realidade psicológica dos modelos. Eles estão interessados numa versão mais abstrata da AI. Para tais pesquisadores é de pouca relevância a questão de se os processos empregados pela máquina na execução das tarefas são semelhantes ou não àqueles utilizados pelos seres humanos.

Em outras palavras, estes últimos pesquisadores preocupam-se apenas em imitar aquilo que a mente pode fazer, deixando de lado os processos através dos quais tais tarefas são realizadas. A simulação cognitiva, ao contrário, tenta imitar a mente e objetiva simular processos mentais que atuam no pensamento e na ação humana.

Ainda que existam diferenças entre as duas vertentes da AI, é comum a todos os seus pesquisadores a mesma tática de análise dos processos responsáveis pelo comportamento inteligente*. Essa tática consiste em elaborar um sistema particular que possa realizar determinada tarefa e considerar, a seguir, quais aspectos desse sistema seriam necessários para que um sistema qualquer executasse a mesma tarefa. A consideração a ser feita tem como pressuposto a tese de que os macro-processos manifestos no comportamento inteligente podem ser devidamente explicados reduzindo-os a mecanismos simples, átomos hipotéticos chamados "Processos Elementares de Informação". Os sistemas que manipulam tais processos são conhecidos em AI como Sistemas de Processamento de Informação (abreviadamente IPS, do inglês Information Processing System).

Uma ilustração da estrutura geral de um IPS é fornecida por A. Newell, H. Simon em seu livro Human Problem Solving** (vide p. 47).

Este sistema é um dispositivo extremamente geral de manipulação de símbolos. Os sinais de símbolos estocados na memória são arranjados em estruturas através de relações que possibilitam a elaboração de listas, com as quais o sistema trabalha.

* Por inteligência entenda-se a "habilidade de um sistema para manipular símbolos ou processos de informação, dadas as exigências da tarefa em questão". M. Boden, Artificial Intelligence and Natural Man, (I, p. 17).

** Newell, A. \& Simon, H. - Human Problem Solving, (11, p. 20). 
TEIXEIRA, J. de F. \& QUILICI GONZALES, M.E. - Inteligência Artificial e teoria de resolução de problemas. Trans/Form/Ação, São Paulo, 6: 45-52, 1983.

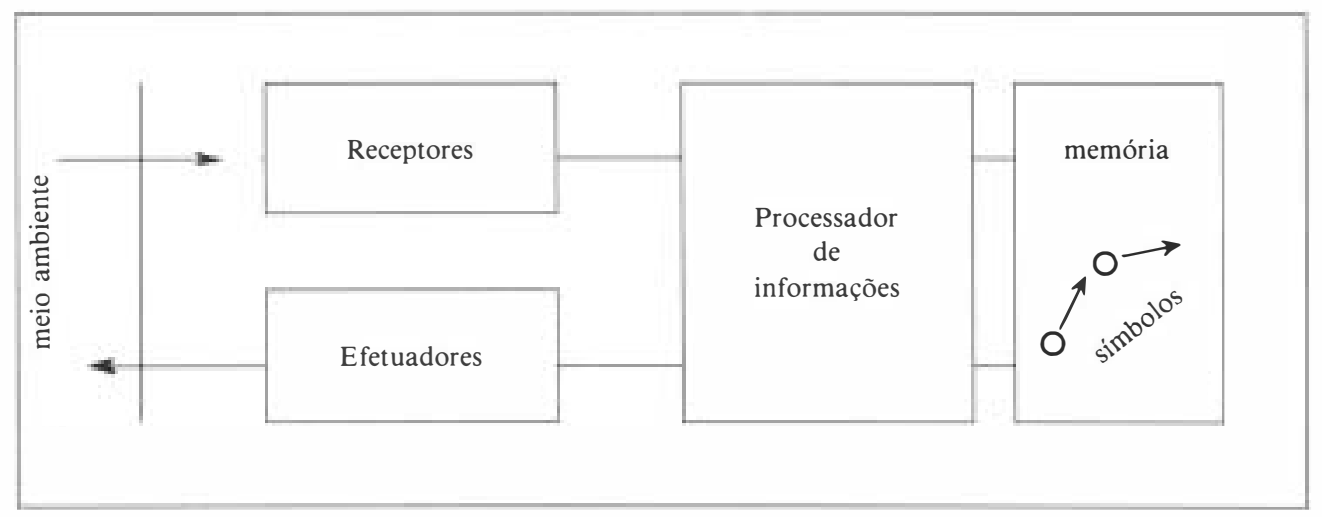

O processador de informações é um componente do IPS que executa todo e qualquer tipo de processo para o qual ele foi construído. O processador consiste de: a - um conjunto fixo de processos elementares de informação,

b - uma memória imediata (short-term) e c - um intérprete.

Os processos elementares de informação, por sua vez, são postulados de acordo com cada sistema particular. Eles não são explicados (isto é, não se fornece uma fundamentação para eles em termos de estruturas ainda mais elementares), mas devem ser bem definidos de forma a poderem ser manipulados por mecanismos conhecidos. Os principais processos elementares de informação disponíveis são aqueles que comparam símbolos, efetuam associações, lêem, copiam e modificam símbolos e estruturas de símbolos. As estruturas compostas, isto é, as listas, são discriminadas umas das outras comparando-se processos que aplicam testes básicos para identificar símbolos em determinadas posições nas estruturas.

Finalmente, a consideração do meio ambiente é de fundamental importância para o funcionamento de um sistema de processamento de informações. O meio ambiente pode ser entendido como a informação disponível para a realização de uma tarefa. Ele atua como base de referência em relação à qual está voltada a meta do siste- ma. Pelo fato de que a delimitação do meio ambiente depende essencialmente da tarefa a ser executada, ele é conhecido como meio ambiente de tarefas.

Levando em conta todos estes elementos, os pesquisadores da AI elaboram programas para a realização de tarefas. Tais programas constituem seqüências hierárquicas e condicionalmente ramificadas de processos elementares de informação. De modo a executar uma tarefa, o IPS efetua uma seqüência de ações na tentativa de transformar a situação inicial " $A$ " que lhe é fornecida, em uma situação desejada "B". A forma em que essa seqüência de "ações" é efetuada pelo programa depende do método de busca estabelecido pelo programador.

A seguir faremos um breve comentário dos métodos de resolução de problemas estudados por Newell, Simon e outros partidários do Projeto de Simulação Cognitiva. A análise desses métodos é relevante, não apenas para o psicólogo, mas também para o filósofo da ciência, na medida em que este admita que o estudo empírico dos métodos de inferência usados pelos indivíduos na tentativa de resolver problemas pode constituir um prelúdio útil ao estudo dos métodos racionais de resolução de problemas. 
TEIXEIRA, J. de F. \& QUILICI GONZALES, M.E. - Inteligência Artificial e teoria de resolução de problemas. Trans/Form/Ação, São Paulo, 6: 45-52, 1983.

\section{A Teoria de Resolução Humana de Pro- blemas}

Com base no estudo do comportamento de indivíduos que resolvem problemas formais simples em laboratório, Newell e Simon construíram alguns programas para simular aspectos do comportamento inteligente. A técnica por eles usada na elaboração dos programas pode ser resumida em poucas etapas: a primeira etapa consiste em gravar as declarações proferidas por alguns indivíduos que verbalizam seu pensamento enquanto resolvem os problemas. A seguir, o teórico ensaia algumas hipóteses acerca dos processos mentais que possivelmente estariam envolvidos na ação em questão. A partir dessas hipóteses ele estrutura um programa que, em sua opinião, simulará o relato gravado. Finalmente, após processar esse programa no computador digital, ele compara o relato do indivíduo com o roteiro da máquina. Se os fluxos de palavras registrados no roteiro e no relatório forem razoavelmente semelhantes, então considera-se que uma explicação para o comportamento sob estudo foi obtida. Os pesquisadores do Projeto de Simulação Cognitiva admitem, neste caso, que as estratégias utilizadas pelo computador são análogas àquelas empregadas pelo indivíduo humano. Caso contrário, o programa deverá ser modificado com base nas discrepâncias encontradas durante o confronto de palavras. O mesmo procedimento é repetido até que um ajuste satisfatório seja obtido e o programa consiga passar pelo teste de Turing. Ou seja, até que os fluxos de palavras produzidas pelo computador e pelo sujeito humano sejam praticamente indistingüíveis para um examinador humano.

As experiências feitas por Newell e Simon revelaram que os indivíduos empregavam, em geral, estratégias comuns durante a tentativa de resolução de problemas, as quais, muitas vezes, conduziam à solução desejada. Estas estratégias foram incorporadas em vários programas para a resolução de problemas, entre eles o conhecido "Solucionador Geral de Problemas" GPS
(General Problem Solver) e o Teórico da Lógica LT (Logic Theorist).

Considerando que temos um problema quando queremos alguma coisa e não sabemos que seqüências de ações executar para alcancá-la, Newell e Simon estruturaram o GPS e o LT de forma a capacitá-los a buscar as seqüências adequadas de ações que poderiam conduzir à solução de problemas. O LT, por exemplo, foi criado para demonstrar teoremas do Cálculo Proposicional. A tarefa de descobrir uma prova consiste em encontrar uma seqüência de operadores as regras do Cálculo - que transforme os objetos inicialmente dados (os teoremas do Cálculo) no objeto-meta, ou seja, o teorema a ser demonstrado. De modo a resolver este tipo de problema, o LT divide a tarefa em outras tarefas que consistem em: 1) Aplicar um operador a um objeto; 2) Reduzir a diferença entre dois objetos, modificando o primeiro dêles;

3) Avaliar a diferença existente entre o objeto transformado e a meta a ser atingida.

À medida que um operador é aplicado a um objeto para reduzir as diferenças existentes entre ele e a meta, novas submetas serão geradas, sendo que estas últimas, uma vez realizadas, podem conduzir à resolução do problema original. Este método de resolver um problema subdividindo-o em problemas mais simples - através da constante comparação entre aquilo que se dispõe e a meta desejada - é chamado "Método de Análise de Meios e Fins". Diferentemente do método exaustivo de tentativa e erro, que sempre conduz a uma solução quando ela existe, o Método de Análise é um método falível. O conjunto de táticas que ele reúne não assegura que a solução do problema será sempre encontrada. Ele apenas sugere como selecionar a informação disponível no meio ambiente de tarefas de tal modo que, ao final do processo, existe grande chance de que o problema seja resolvido. Este método é igualmente empregado pelo GPS que reúne também outros métodos, tais como o Método de Planejamento. O Método de Planejamento consiste em abstrair os detalhes dos 
TEIXEIRA, J. de F. \& QUILICI GONZALES, M.E. - Inteligência Artificial e teoria de resolução de problemas. Trans/Form/Ação, São Paulo, 6: 45-52, 1983.

objetos analisados de modo a reduzir as diferenças existentes, não entre os próprios objetos, mas entre os objetos mais abstratos dos quais os primeiros são apenas instanciações.

Através do emprego dos Métodos de Análise, de Planejamento e de outros métodos, o GPS seleciona a informação relevante para a execução de uma determinada tarefa sem levar em conta o restante da informação que, embora disponível no meio ambiente, não esteja vinculada à meta do problema a ser solucionado. Esse programa recebeu o nome de "Solucionador Geral de Problemas" porque a manipulação desses métodos permite resolver diferentes tipos de problemas, sejam eles problemas de xadrez, de Lógica Elementar, etc.

Os autores do GPS e do LT admitem que a evidência fornecida pelos vários experimentos realizados empregando a técnica de simulação é suficiente para verificar a hipótese de que as estratégias utilizadas pelo computador e pelo sujeito humano são as mesmas. Com base nesta suposição, e tendo claramente especificadas as estratégias utilizadas pelos indivíduos, Newell e Simon desenvolveram a Teoria de Resolução Humana de Problemas. Para eles, "as teorias explicam o comportamento manifesto na execução de uma tarefa à medida em que elas descrevem a manipulação da informação num nível elementar, onde um intérprete simples (tal como um computador digital) pode transformar a descrição num processo efetivo para realizar sua meta". *

As idéias de Newell e Simon no que diz respeito a explicação do comportamento humano inteligente através do computador digital enfrentam várias dificuldades. Os próprios autores concordam que elas constituem apenas a etapa inicial de um programa de pesquisa a ser desenvolvido. Ainda que atualmente grande progresso tenha sido feito nesta área, a qual foi subdividida em várias subáreas de estudo, a importância histórica das idéias de Newell e Simon é inegável. Desde sua formulação no arti- go "The Simulation of Human Thought" muitas críticas têm sido elaboradas, especialmente por autores tais como H. Dreyfus (3), K. Gunderson (6), J. Haugeland (7) e outros. Algumas dessas críticas serão abordadas a seguir. Elas sugerem várias questões que permitem aproximar a investigação em Inteligência Artificial com a investigação empreendida pela Psicologia e por certos ramos da Filosofia, em especial a chamada Filosofia da Mente.

\section{Filosofia, Epistemologia e Simulação Cognitiva}

Entre as várias questões formuladas pelos críticos da AI, encontram-se aquelas levantadas por J. Fodor num artigo de 1965 intitulado "The Logic of Simulation", posteriormente incorporado ao livro Psycological Explanation(4) (1968). Ao discutir o Projeto de Simulação Cognitiva, Fodor sugere que este tipo de empreendimento encontra um de seus pontos fracos no fato de que o comportamento simulado mecanicamente não é o resultado de uma escolha dentre um repertório de comportamentos possíveis como ele julga ocorrer no caso do ser humano.

Um segundo problema, também assinalado por Fodor, diz respeito à possibilidade de se afirmar que realmente duplicamos não apenas os comportamentos manifestos mas também os processos a eles subjacentes. Esta afirmação envolve questões complexas, dando lugar a um problema parecido com o problema da adequação de teorias a dados empíricos selecionados para confirmá-las.

Se estendemos as críticas de Fodor aos experimentos realizados por Newell e Simon e codificados no GPS e no LT, verificaremos que eles enfrentam o mesmo tipo de problemas. Com ęfeito, podemos imediatamente questionar se os nossos processos cognitivos subjacentes à atividade de resolução de problemas são semelhantes às táticas empregadas por tais programas, tais como o Método de Análise de Meios e Fins

\footnotetext{
${ }^{*}$ Newell, A. \& Simon, H. - Human Problem Solving, (11, p. 11).
} 
TEIXEIRA, J. de F. \& QUILICI GONZALES, M.E. - Inteligência Artificial e teoria de resolução de problemas. Trans/Form/Ação, São Paulo, 6: 45-52, 1983.

e o Método de Planejamento. Sempre é possível pensar que podem existir outros tipos de táticas para resolver problemas e, em especial, ef etuar demonstrações no domínio mesmo do Cálculo Proposicional. Hao Wang (17), por exemplo, propõe um outro conjunto de estratégias para solucionar problemas no Cálculo Proposicional. O programa montado por Wang não se utiliza de estratégias heurísticas tais como aquelas propostas por Newell e Simon. As provas para teoremas do Cálculo Profissional são obtidas através de um método chamado Método Algorítmico. A diferença consiste no fato de que o método de Wang se utiliza das chamadas tabelas de verdade, constituindo-se num procedimento infalível de prova, na medida em que ele percorre todos os casos possíveis numa demonstração. A pergunta que se coloca então é a seguinte: pode o método de Wang ser considerado uma simulação dos nossos processos cognitivos? Como decidir qual dos dois programas constitui uma simulação do processo humano de resolver problemas do Cálculo Proposicional? Como poderíamos negar que os seres humanos se utilizam de procedimentos algorítmicos em vez de heurísticos? Ao que tudo indica, não dispomos de instrumentos para decidir entre uma simulação e outra, uma vez que ambas se baseiam em resultados manifestos e nada nos impede de atribuir aos processos cognitivos humanos uma extraordinária velocidade que permitiria afirmar que tais procedimentos são igualmente bem desempenhados algoritmicamente. *

Problemas como este podem levar-nos a colocar em questão a validade do Teste de Turing, a que nos referimos no início deste artigo, como critério de decisão para avaliar a "performance" de programas que tentam imitar atividades humanas inteligentes. Por outro lado, os problemas enfrentados pelo Projeto de Simulação Cognitiva não parecem se esgotar aí. Um outro tipo de questão pode também ser levan- tado: será que é possível estipular - como pressupõem Newell e Simon - que existe uma similaridade entre a atividade mental dos sujeitos e o seu comportamento lingüístico manifesto? A pressuposição dessa similaridade é bastante questionável, e conquanto possamos admitir, num primeiro momento, que a linguagem é parte constitutiva (e essencial) dos nossos processos mentais, isto não permite descartar a hipótese de que processos lingüísticos são apenas manifestações de operações mais profundas.

No seu livro The Language of Thought (5)Fodor estuda um conjunto de atividades cognitivas que parecem ser reveladoras da existência de estruturas mais profundas do que a linguagem natural. Não nos cabe aqui reproduzir os experimentos relatados por ele, dada a sua extensão. De qualquer maneira, esta questão permanece.

Se se estipula a existência destas estruturas mais profundas, o que pensar dos experimentos de simulação cognitiva? Seríamos por acaso forçados a abandoná-los por questões de princípio que obstariam sua realização? A isto respondemos negativamente, pois, se por um lado verificamos que a duplicação de processos cognitivos através de equivalentes mecânicos é dificilmente exeqüível, isto não quer dizer que a simulação cognitiva não tenha utilidade como estratégia de investigação.

Ao tentar criar equivalentes mecânicos de processos cognitivos, o investigador da AI é obrigado a elaborar programas, e nesta atividade ele tem de estabelecer, com clareza, cada etapa a ser percorrida pelo computador. Nesta tarefa, ele é simultaneamente obrigado a uma reflexão mais detalhada acerca de suas hipóteses sobre a estrutura e o funcionamento das atividades cognitivas humanas. Tudo se passa, como diz D. Dennett "como se montar programas equivalesse a esquematizar diagramas no quadro-negro numa aula de geometria"**. Teo-

* Este mesmo exemplo é discutido por K. Gunderson (6).

** A referência é ao livro de D. Dennett (2, p. 117-118). 
TEIXEIRA, J. de F. \& QUILICI GONZALES, M.E. - Inteligência Artificial e teoria de resolução de problemas. Trans/Form/Ação, São Paulo, 6: 45-52, 1983.

ricamente, eles podem ser considerados irrelavantes, mas, por outro lado, eles forçam uma certa disciplina de imaginação. Ademais, o investigador é obrigado a se levantar questões concernentes à natureza do comportamento humano, problemas relativos ao reconhecimento de padrões (pattern-recognition), etc. Enfim, a investigação em AI cruza-se com a investigação filosófica na medida em que construir programas significa muitas vezes indagar pelas condições de possibilidade de certos desempenhos cognitivos presentes na linguagem natural, na percepção, etc.

Uma outra questão, que tem chamado a atenção de filósofos e psicólogos*, consiste em saber como um organismo pode construir suas representações internas. $\mathrm{O}$ conceito de representação interna tem se mostrado fundamental na explicação do comportamento para várias escolas psicológicas. É a existência de representações in- ternas que nos permite atribuir "subjetividade" a organismos.

Uma importante vertente da investigação em AI tem se dedicado ao estudo do funcionamento das representações internas através da montagem de programas. As representações internas atuam nos organismos como uma espécie de modelo interno do mundo, ou mesmo como modelos hipotéticos do mundo. Elas são, em geral, alojadas no nosso sistema nervoso, influenciando nossa ação corporal. Contudo, sua base fisiólogica é ainda bastante obscura, especialmente quando elas funcionam como representações epistemológicas do mundo. $\mathrm{O}$ estudo e a descrição destas representações através de computadores, a possibilidade de construir programas nos quais esteja embutida uma simulação deste tipo de atividade é uma tarefa que vem sucessivamente aproximando a AI da Psicologia e da Filosofia, em especial da Teoria do Conhecimento. Sobre isto falaremos num outro artigo.

TEIXEIRA, J. de F. \& QUILICI GONZALES, M.E. - Artificial Intelligence and the problem solving theory. Trans/Form/Ação, São Paulọ, 6: 45-52, 1983.

ABSTRACT: This article is an attempt to outline the main characteristics of research on a new area of studies, the so-called Artificial Intelligence (AI). Items 1 and 2 sketch the historical background of AI and its basic pressuppositions. Item 3 focus on the problem-solving theory developed by $A$. Newell and $H$. Simon. Item 4 is an attempt to show the philosophical and epistemological relevance of Artificial Intelligence. lation.

KEY-WORDS: Artificial intelligence; philosophy of mind; problem-solving-methods; cognitive simu-

\section{REFERÊNCIAS BIBLIOGRÁFICAS}

1. BODEN, M. - Artificial Intelligence and natural man. Brighton, Sussex, Harvester Press, 1977.

2. DENNETT, D. - Brainstorms. S.l., Bradford Books, 1978.

3. DREYFUS, H. - What computers can not do. New York, Harper \& Row, 1972.
4. FODOR, J. - Psychological explanation. New York, Randon House, 1968.

5. FODOR, J. - The language of thought. Sussex, The Harvester Press, 1976.

6. GUNDERSON, K. - Mentality and machines. New York, Anchor Books, 1971.

7. HAUGELAND, J. - Mind design. Vermont, Bradford Books, 1981.

* A referência é ao livro de D. Dennett (2, p. 117-118). 
TEIXEIRA, J. de F. \& QUILICI GONZALES, M.E. - Inteligência Artificial e teoria de resolução de problemas. Trans/Form/Ação, São Paulo, 6: 45-52, 1983.

8. MUller, G.A. \& PRIBRAM, J. - Plans and the structure of behavior. New York, Holt, 1960.

9. NEWELL, A. \& SIMON, H. - GPS a program that simulates human thought. In: FEINGEMBAUM \& FELDMAN, eds. - Computers and throught. New York, McGraw-Hill, 1968. p. 279-293.

10. NEWELL, A \& SIMON, H. - The simulation of human thought. Current Trends in Psychological Theory, no. 1: 152-179, 1961.

11. NEWELL, A. \& SIMON, H. - Human problem solving. New York, Prentice Hall, 1972.

12. NILSSON, N. - Problem-solving methods in Artifical Intelligence. New York, McGraw Hill, 1971.
13. SIMON, H. - Models of discovery. Cambridge, Mass. MIT Press, 1974.

14. SIMON, H. - The science of artificial. Cambridge, Mass. MIT Press, 1978.

15. SLOMAN, A. - The computer revolution in philosophy. Sussex, Harvester Press, 1978.

16. TURING, A. - Computing machinery and intelligence. In: FEINGEMBAUM \& FELDMAN, eds. - Computers and thought. New York, McGraw-Hill, 1968. p. 11-35.

17. WANG, H. - Toward mechanical mathematics. IBM Journal of Research and Development, n. $4: 2-22,1960$. 